\title{
Author Index for Volume 63
}

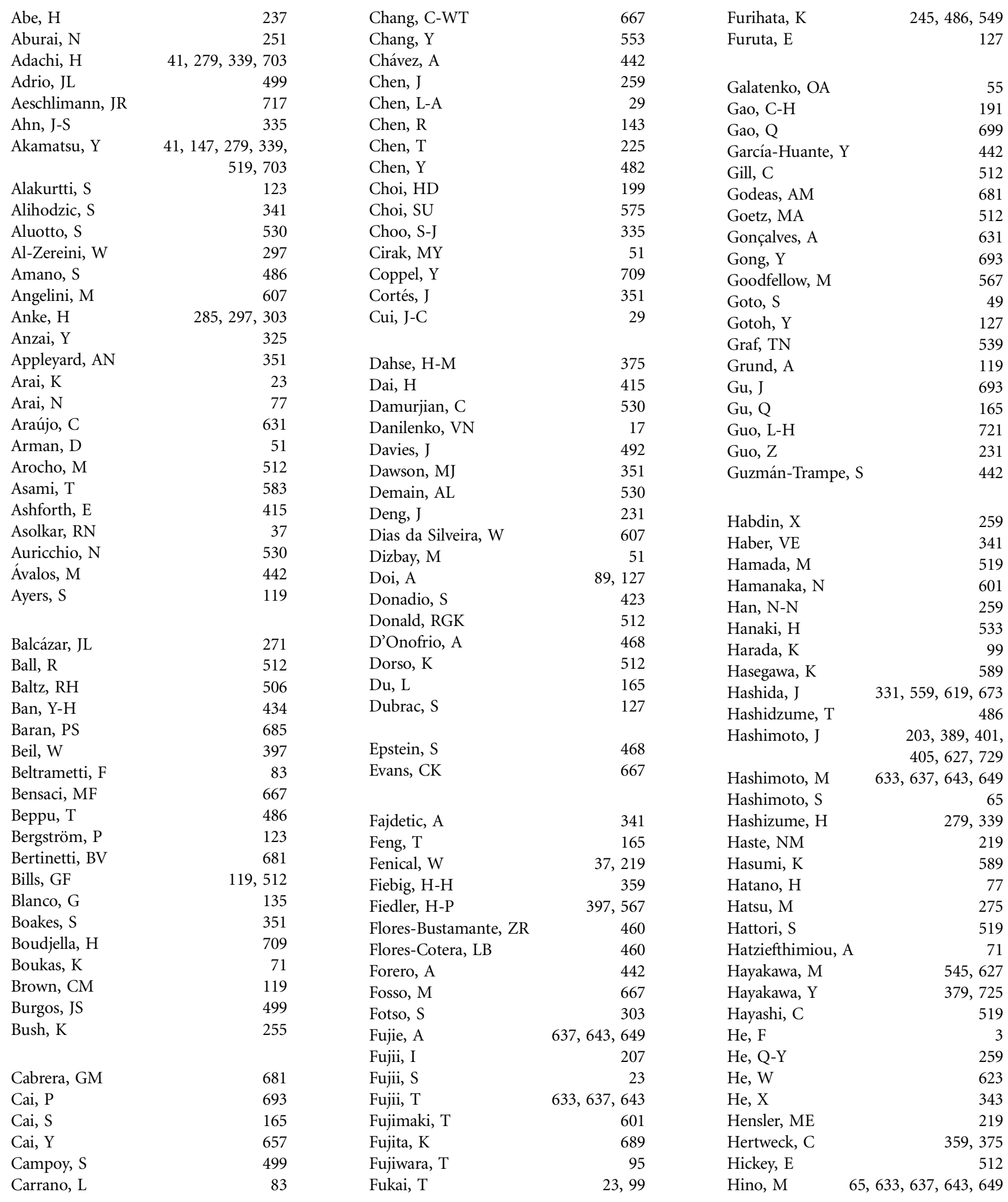




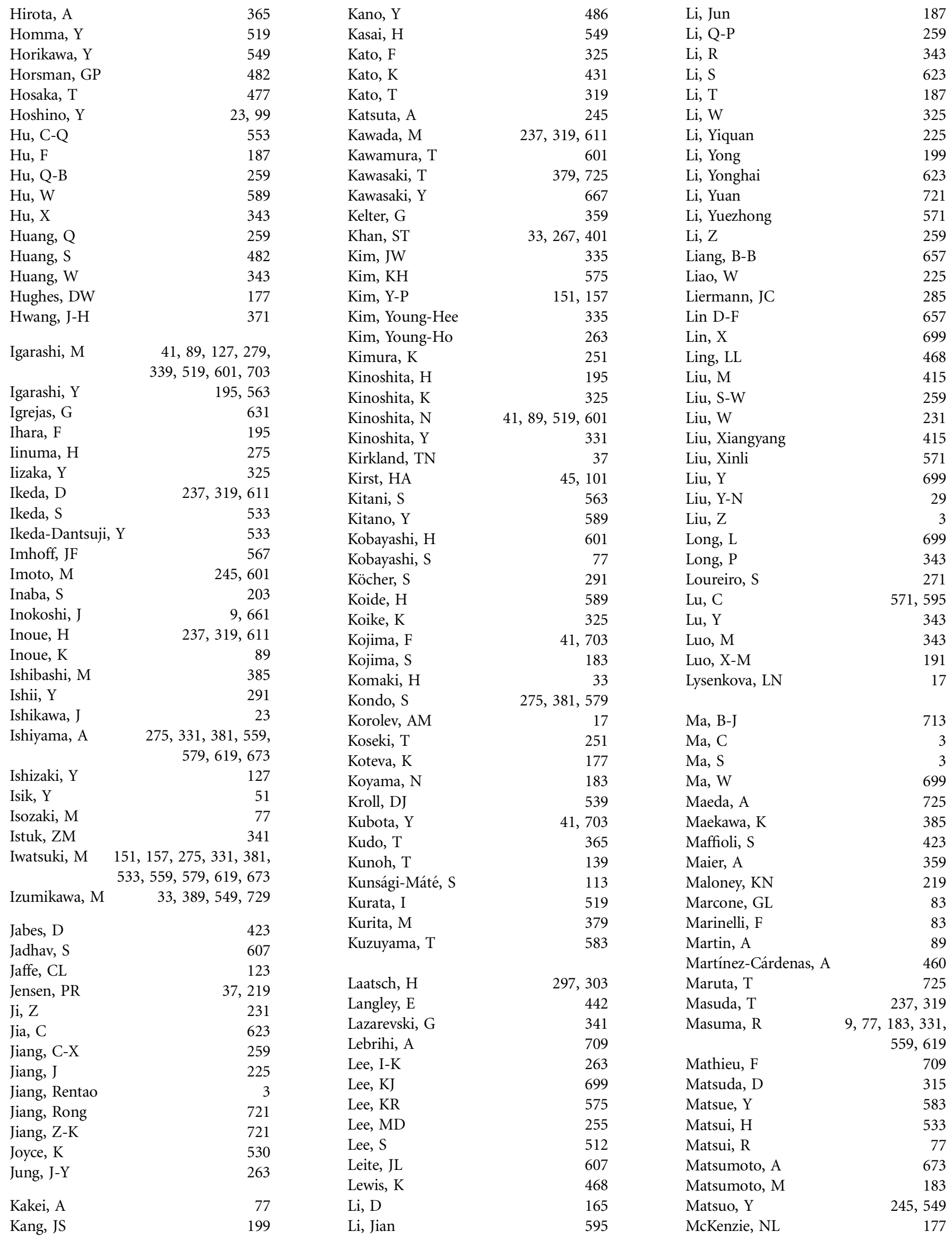




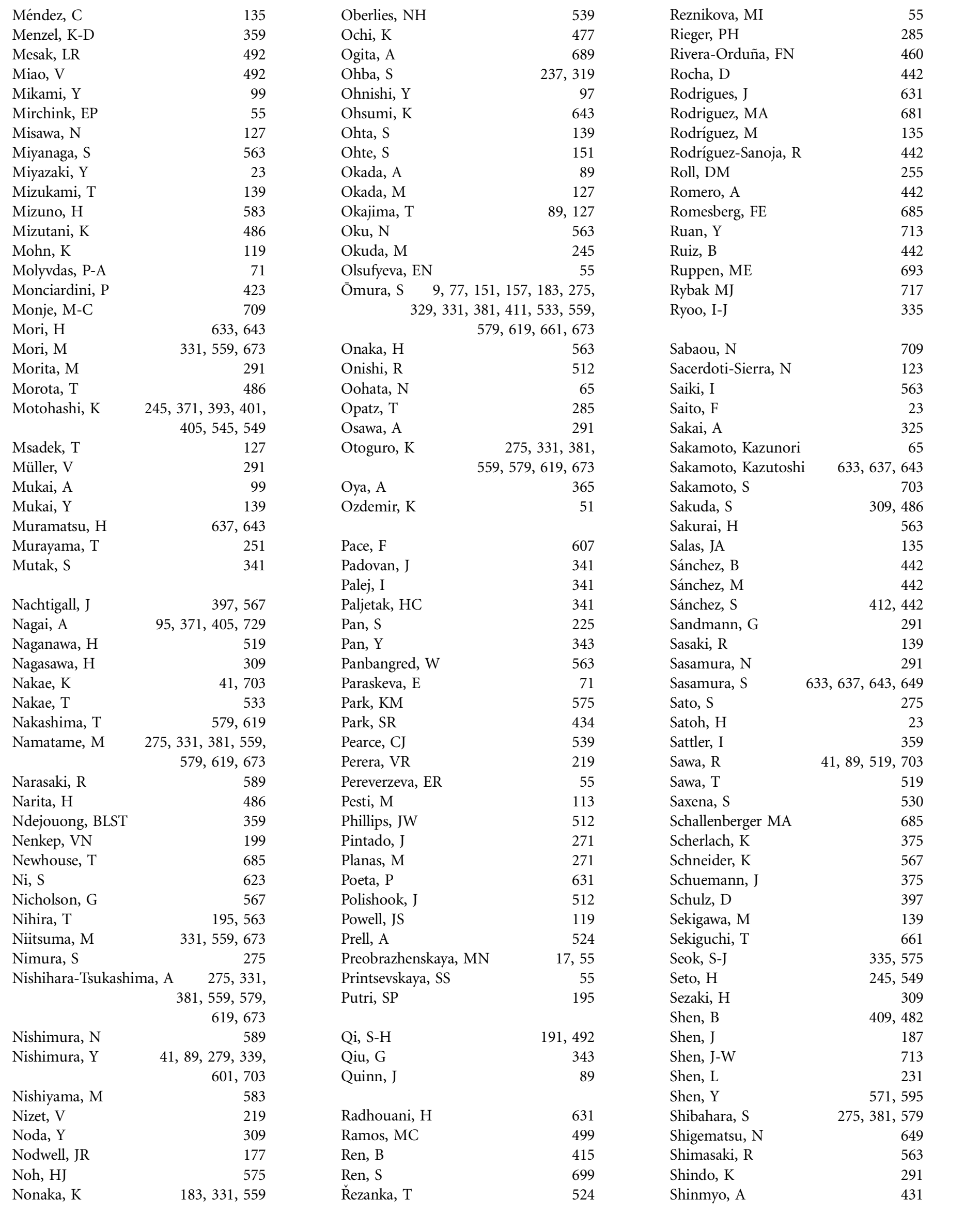


Shinonaga, $\mathrm{H} 23$

Shin-ya, K 33, 95, 139, 203, 267, 371, $379,389,393,401,405$, $545,549,615,627,729$

Shiomi, K 77, 331, 533, 559, 673

Shiono, Y 251

Shirai, M 245

Shizuri, Y 245, 549

Shrestha, S 667

Si, S-Y 259

Sierra, S 499

Sigler, K 524

Silva, N 631

Silva, YJD 271

Singh, SB 119, 512

Skwish, S

Solovieva, SE

512

Someno, T

Son, BW

Song, F

Song, $\mathrm{H}$

Song, X-J

Sosio, $\mathrm{M}$

Spížek, J

Stamatiou, R

Stehling, EG

Stimac, V

Storz, T

Su, X

Suarez, B

Sue, M

Süssmuth, RD

Sugiyama, $\mathrm{Y}$

Sun, C-H

Sunakawa, K

Suzuki, K

Sy-Cordero, AA

237, 319, 611

199

415

3

657

423

524

71

607

341

693

225

499

549

397, 567

365

143, 259, 721

533

661

539

Tabata, Y

237

Takagaki, S

633

Takagi, M 33, 95, 139, 203, 267, 371, $379,389,393,401,405$, $545,549,615,627,729$

Takagi, Y

Takahashi, A

661

Takahashi, Yoko

619, 673

Takahashi, Yoshikazu

$147,519,601$

Takano, H

Takano-Shiratori, $\mathrm{H}$

Takase, S

486

486

Takemoto, JY

$633,637,643$

Takenaka, A

667

661

Tanaka, $\mathrm{H}$

Tanaka, T

77,661

689

Tanaka, Y

477

Tao, L

259

Tashiro, E

601

Tavcar, B

341

Tevyashova, AN

55

Thaker, M

177

Thines, E
Thompson, D

Tian, X-P

Toda, $\mathrm{T}$

Tohyama, S

Tomita, T

Tomoda, H

Torii, K

Toume, K

Tozlu, DK

Tran, DN

Trenin, AS

Treshalin, ID

Tsuboi, M

Tsunoda, M

Turberg, A

Turchin, KF

Tynan, $\mathrm{H}$

Uchida, $\mathrm{R}$

Ueda, J

Ueda, K

Ueda, S

Ueno, $\mathrm{M}$

Ugaki, N

Ui, $\mathrm{H}$

Umekita, M

Usami, I

Utsumi, R

Velasco, J

Vicente, $\mathrm{F}$

Vidaillac, C

Villanueva, I

Virág, E

Wada, S

Wang, F

Wang, $\mathrm{H}$

Wang, Jian

Wang, Jinhua

Wang, J-D

Wang, Ming

Wang, Minggui

Wang, $\mathrm{P}$

Wang, R

Wang, X-J

Wang, Yiguang

Wang, Yong-Hong

Wang, Yucheng

Wani, MC

Watanabe, Takafumi

Watanabe, Takumi

Wei, $\mathrm{X}$

Wei, Y-Z

Wildey, MJ

Williams, A

Wimonsiravude, W

Wright, GD

$\mathrm{Wu}, \mathrm{J}-\mathrm{B}$

$\mathrm{Wu}, \mathrm{L}$
Wu T-T

713

Wu, W

231

$\mathrm{Wu}, \mathrm{Z}$

171

Xiang, W-S

165

9, 151, 157, 183,

315,329

601

385

51

219

55

55

65

661

77

17

530

9, 151, 157

$139,203,267,615,627$

486

65

649

315

559

$89,519,703$

237

89,127

499

512

717

492

113

139

165

623

415

667

171

171

187

191

657

171

623

553

623

539

89

237, 519

225

259

255

530

563

177

721

623

Xiao, $\mathrm{X}$

Xiao, Y

343

Xie, Q

415

Xie, Y

143

Xiong, $\mathrm{Z}$

187

$\mathrm{Xu}, \mathrm{G}-\mathrm{H}$

$\mathrm{Xu}, \mathrm{H}$

$\mathrm{Xu}, \mathrm{T} \quad 699$

Xu, Yinya 343

$\mathrm{Xu}$, Yunjian 225

Yamada, H

275, 331, 381, 559,

Yamada, M

$579,619,673$

Yamada, $T$

65

Yamada, Y

633,643

Yamaguchi, S

725

Yamaguchi, Y

237, 703

Yamamoto, S

77

Yamamura, $\mathrm{H}$

486

Yamashita, Michio

545,627

Yamashita, Midori

65

Yamazaki, $\mathrm{H}$

649

Yamazaki, Y

315,329

Yan, L-L

379

259

Yanagisawa, C

533

Yang, B

699

343

Yang, J

259

Yang, X-W

699

Yang, $\mathrm{Y}$

255

Yao, FF

297, 303

Yazawa, K

99

Yi, J

225

Yin, $\mathrm{H}$

699

Yin, $\mathrm{M}$

482

Yli-Kauhaluoma, J 123

Yoda, K

309

Yokoi, M

251

Yoo, I-D

335

Yoo, Y

434

434

Yoshikawa, H 127 
Zhang, J-P

Zhang, L

Zhang, S

Zhang, X-D

Zhang, Y

Zhang, Y-Q
Zhang, Z-Z

Zhao, B

Zhao, X

Zheng, G-H

Zhou, $\mathrm{H}$

Zhou, X-F
Zhu, T

165

165

713

259

713

699
Zinecker, $\mathrm{H}$

Zink, DL

Zitouni, A

Zotchev, SB
567

119, 512

709 